\title{
A Polynomial Digital Pre-Distortion Technique Based on Iterative Architecture
}

\author{
Kwang-Pyo Lee, Soon-Il Hong, Eui-Rim Jeong* \\ Department of Information and Communication Engineering, National Han Bat University, 305-719, Korea
}

\begin{tabular}{l} 
Article Info \\
\hline Article history: \\
Received Aug 20, 2015 \\
Revised Nov 5, 2015 \\
Accepted Nov 22, 2015 \\
\hline Keyword: \\
Digital pre-distortion \\
Indirect learning \\
Iterative structure \\
polynomial \\
Power amplifiers (PAs)
\end{tabular}

\begin{abstract}
A digital predistortion (DPD) technique based on an iterative adaptation structure is proposed for linearizing power amplifiers (PAs). To obtain proper DPD parameters, a feedback path that converts the PA's output to a baseband signal is required, and memory is also needed to store the baseband feedback signals. DPD parameters are usually found by an adaptive algorithm by using the transmitted signals and the corresponding feedback signals. However, for the adaptive algorithm to converge to a reliable solution, long feedback samples are required, which increases hardware complexity and cost. Considering that the convergence time of the adaptive algorithm highly depends on the initial condition, we propose a DPD technique that requires relatively shorter feedback samples. Specifically, the proposed DPD iteratively utilizes the short feedback samples in memory while keeping and using the DPD parameters found at the former iteration as the initial condition at the next iteration. Computer simulation shows that the proposed technique performs better than the conventional technique, as the former requires much shorter feedback memory than the latter.
\end{abstract}

Copyright (C) 2016 Institute of Advanced Engineering and Science. All rights reserved.

\section{Corresponding Author:}

Eui-Rim Jeong, Department of Information and Communication Engineering, Hanbat National University 125 Dongseodaero, Yuseong-gu, Daejeon, 305-719, Korea, Email: erjeong@hanbat.ac.kr

\section{INTRODUCTION}

A power amplifier (PA) is an essential component to transmit signals to a remote destination in wireless communications. Unfortunately, the PA is the major source of nonlinearities in a wireless communication system, resulting not only in spectral regrowth, namely strong interference for the adjacent communication channels, but also in-band distortions, if it is driven close to the saturation point [1]. In order to overcome those problems caused by nonlinearity, the PA is generally backed off to operate within the linear portion of its operating curve. However, when operated at a lower power than the saturation power of the PA, it will have low power-efficiency around 10\%. Thus, various linearization techniques have been proposed to achieve high power-efficiency of the PA [2]. Among these techniques, digital pre-distortion is known to be the most cost- and performance-effective technique. It adds a predistorter (PD) in the baseband digital stage to create a distorted signal that is complementary to the compressing characteristic of the PA. A typical DPD system, as shown in Figure 1 consists of digital predistorter (DPD), DPD engine, DAC (digitalto-analog converter), ADC (analog-to-digital converter), upconverter and downconverter. The transmit signal is pre-distorted by the DPD in the digital stage before it is converted to an analog signal, up-converted to the target RF band, and fed to the PA. In the DPD engine, an adaptive algorithm finds the DPD parameters for the inverse function of the PA, which requires a feedback path to convert the PA's output to a baseband signal and memory to store the feedback signals. By using the parameters, the PD can generate a properly distorted signal for linearizing the PA. However, for the adaptive algorithm to converge to a reliable solution, long feedback samples are required, which increases hardware complexity and cost due to the large memory. 
To solve this difficulty, we propose a DPD technique that requires relatively shorter feedback samples, while the linearization performance is similar to the conventional method with long feedback samples. Specifically, the proposed DPD iteratively utilizes the short feedback samples in memory while keeping and using the DPD parameters found at the former iteration as the initial condition at the next iteration. In general, the adaptive algorithm converges quickly if the initial parameter is close to the solution. At each iteration, the parameter approaches the solution more closely, since the initial parameter becomes close to the solution, as well. Computer simulation shows that the proposed technique performs better than the conventional technique, as the former requires much shorter feedback memory than the latter.

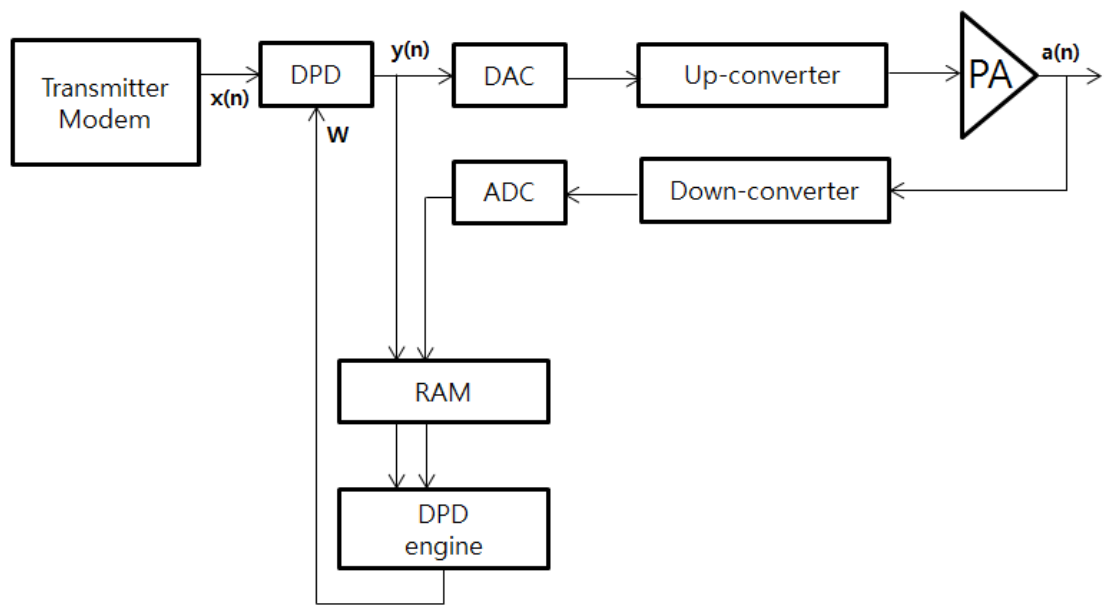

Figure 1. Transmitter architecture employing DPD

\section{DPD STRUCTURE AND SIGNAL MODEL}

Let $y(n)$ and $a(n)$ be the input and output of the PA, respectively, and let $G(\square)$ denotes the PA's characteristic. The PA output can be written as follows:

$$
a(\mathrm{n})=G(y(n))
$$

Similarly, if $x(\mathrm{n})$ and $y(\mathrm{n})$ are the input and output of the PD, respectively, and $F_{\text {pre }}(\square)$ is the PD's characteristic, then the PD output can be written as follows:

$$
\mathrm{y}(\mathrm{n})=F_{\text {pre }}(x(n))
$$

The ideal predistorter should satisfy

$$
a(\mathrm{n})=G\left(F_{\text {pre }}(x(n))\right)=K_{o} x(\mathrm{n})
$$

where $K_{o}$ is the PA's ideal linear gain. We assume that $K_{o}=1$ hereafter. In this paper, a polynomial-based DPD is employed, and its parameters are estimated by an indirect learning method, as shown in Figure 2 [3]. Specifically, the polynomial coefficients are found by linearizing PA - postdistorter, and then the parameters of the postdistorter are copied to the DPD in the Tx path. Denoting $\hat{y}(n)$ as the postdistorter output, it is written as follows:

$$
\hat{y}(n)=F_{\text {post }}\left(\frac{1}{K_{o}} a(n)\right)=\mathbf{w}^{H} \mathbf{a}(n)
$$

where $F_{\text {post }}(\square)$ is the characteristic function of the postdistorter in the feedback path, and 


$$
\begin{aligned}
& \mathrm{w}=\left[w_{0}, w_{1}, \cdots, w_{P}\right]^{T} \\
& \mathrm{a}(\mathrm{n})=\left[\frac{1}{K_{o}} a(n), \frac{1}{K_{o}} a(n)\left|\frac{1}{K_{o}} a(n)\right|^{2}, \cdots, \frac{1}{K_{o}} a(n)\left|\frac{1}{K_{o}} a(n)\right|^{2 P+1}\right]^{T}
\end{aligned}
$$

$2 \mathrm{P}+1$ is the maximum nonlinear order of the postdistorter, and $\mathbf{w}$ is the polynomial coefficient vector of the postdistorter. The predistorter and postdistorter have the same polynomial structure, and their polynomial coefficients are the same, as well. Consequently, the PD output can be expressed as:

$$
\mathrm{y}(\mathrm{n})=x(n) \sum_{p=0}^{P} w_{p}^{*}|x(n)|^{2 p}=\left[w_{0}^{*}, w_{1}^{*}, \ldots, w_{P}^{*}\right]\left[\begin{array}{c}
x(n) \\
\mathrm{x}(\mathrm{n})|x(n)|^{2} \\
\vdots \\
x(\mathrm{n})|x(n)|^{2 P}
\end{array}\right]=\mathbf{w}^{\mathrm{H}} \mathbf{x}(n)
$$

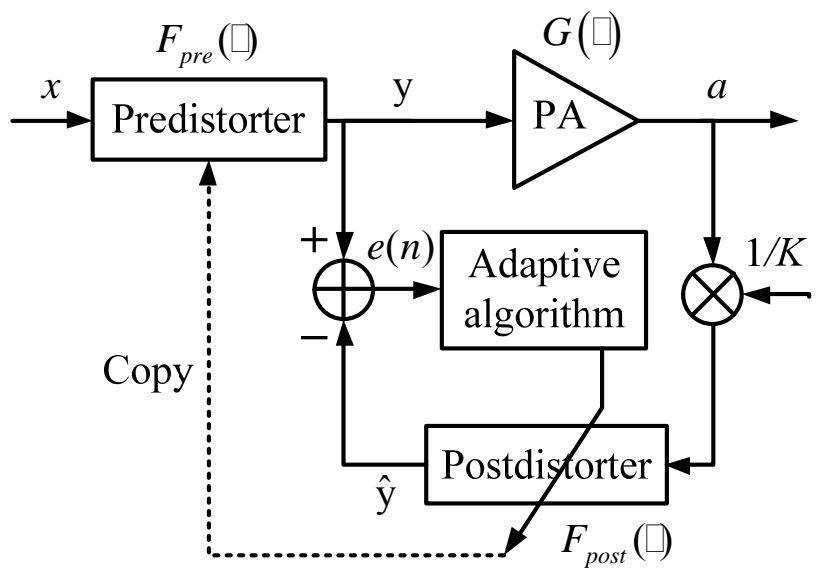

Figure 2. Indirect learning structure

\section{PROPOSED PD ALGORITHM}

To obtain the optimal $\mathbf{w}$ that linearizes the PA, it is generally found via adaptive algorithms, such as the least mean square (LMS) [4] or the recursive least squres (RLS) critertion [5]-[6]. the propsed algorithm was developed based on RLS criterion. The proposed algorithm is presented in Table 1. We denote the transmitted signal (or the PD input) as $x(\mathrm{n})$, the DPD output signal as $\mathrm{y}(\mathrm{n})$, the feedback signal as $\mathrm{a}(\mathrm{n})$, and $\mathbf{w}$ as the DPD coefficient vector. The $(P+1) \times(P+1)$ matrix $\mathbf{Q}$ is defined as the inverse correlation matrix, and the forgetting factor is defined as $\lambda$. First, $\mathbf{w}$ and $\mathbf{Q}$ are initialized with an all-zero vector and the $(P+1) \times(P+1)$ identity matrix, respectively. Assume that the feedback signal length is $N$, i.e., the memory size for storing the feedback signal is $\mathrm{N}$. If $\mathrm{N}$ is not sufficiently large, the adaptive algorithm cannot converge. In the proposed method, however, $\mathbf{w}$ and $\mathbf{Q}$ are found by using the $\mathrm{N}$ feedback samples repetitively. Noting (1) and (2) in Table 1, the coefficient vector $\mathbf{w}$ and the inverse correlation matrix $\mathbf{Q}$ are found for $n=1, \cdots, N$. At $n=N$, both parameters are closer to the optimum solution. However, when the length of the feedback samples, $\mathrm{N}$, is not sufficiently long, it leads to the degradation of the linearization performance. To resolve this problem, the proposed method keeps the last $\mathbf{w}$ and $\mathbf{Q}$ acquired at $n=N$. Then, those parameters become the initial parameters $\mathbf{w}$ and $\mathbf{Q}$, respectively, and the adaptive algorithm is run again with the same feedback samples. This procedure can be repeated several times. Since the RLS algorithm converges more quickly when the initial parameters are closer to the solution, the proposed algorithm utilizes this characteristic to converge the algorithm with short feedback samples. Finally, the PD uses $\mathbf{w}$ obtained at the final iteration. 
Table 1. The Proposed algorithm

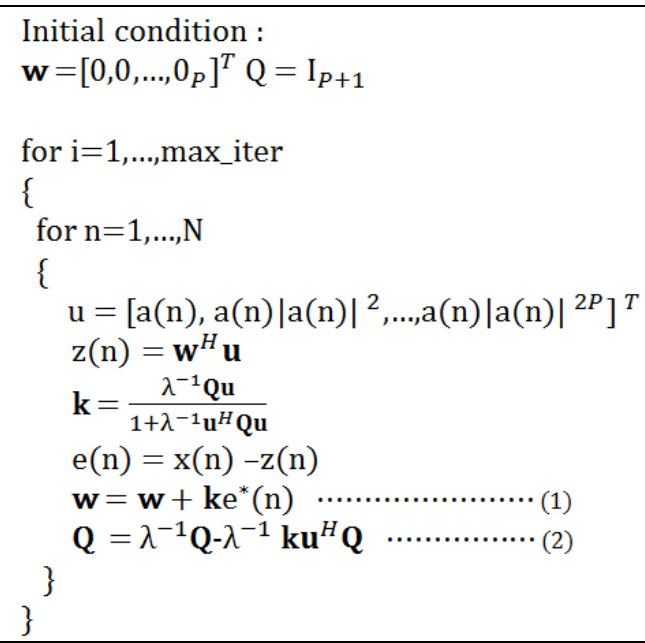

\section{COMPUTER SIMULATION}

To verify the performance of the proposed method, simulation was carried out by using MATLAB. The simulation environments that were used are as follows. The transmitted signal is LTE Downlink signal with $20 \mathrm{MHz}$ bandwidth. The PD's maximum polynomial order is $5(\mathrm{P}=2)$. The forgetting factor $\lambda$ for the RLS algorithm is 0.99999. For the nonlinear PA model, Saleh's model is employed [7], which is given by

$$
\begin{aligned}
& \mathrm{G}(\mathrm{y}(\mathrm{n}))=y(n) \times \frac{K_{1}}{1+K_{2}|y(n)|^{2}} e^{\frac{K_{3}|y(n)|^{2}}{1+K_{4|y(n)|^{2}}}} \\
& K_{1}=1.1, K_{2}=0.3, K_{3}=1, K_{4}=1
\end{aligned}
$$

Figure 3 shows the PA model's AM-AM and AM-PM responses. It is observed that the nonlinear distortion is notable in that the PA input magnitude becomes larger.

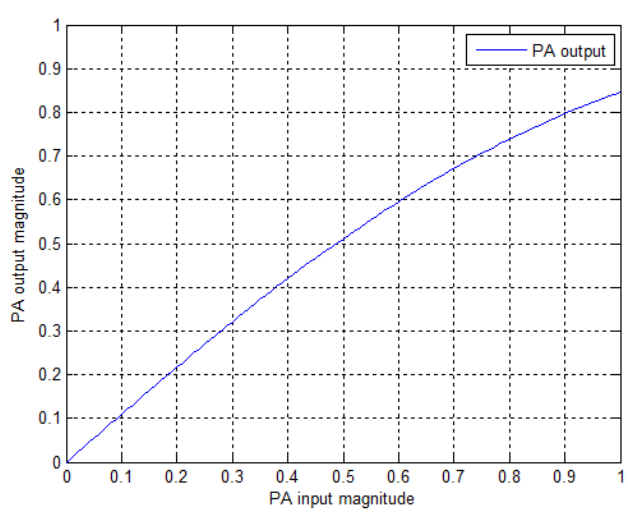

Figure 3. (a) AM/AM response

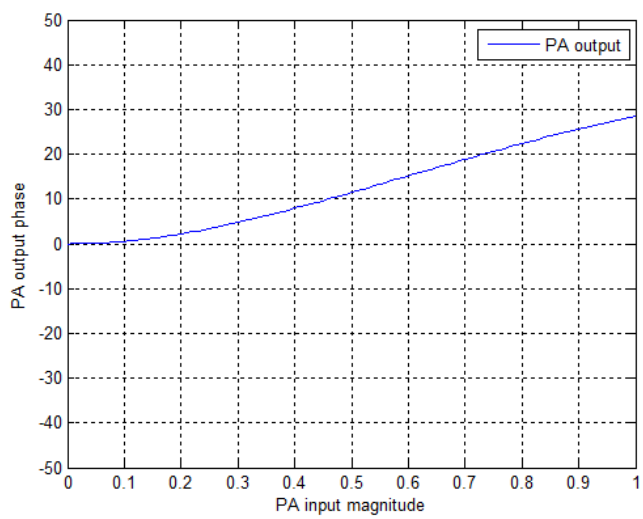

Figure 3. (b) AM/PM response

Figure 4(a) shows the conventional DPD's learning curve for 5,000 feedback samples. The learning curve shows mean square error $(\mathrm{MSE}), \mathrm{E}\left[|\mathrm{e}(\mathrm{n})|^{2}\right]$ in Table I for time $\mathrm{n}$. It is observed that the adaptive algorithm converges to $10^{-4}$ at $n=5,000$. Figure 4(b) shows the learning curve of the proposed method with 10 iterations. At the end of the final iteration, the MSE converges to $10^{-5}$. The MSE is 10-fold better than that of the conventional technique. This result indicates that the DPD parameters are closer to the optimum solution as the number of iterations increases, and better linearization performance is expected by using the proposed technique. 


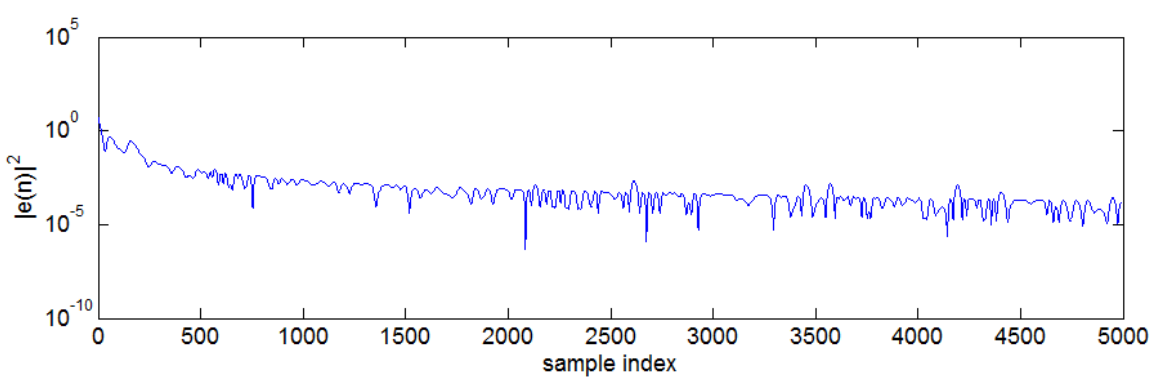

Figure 4. (a) Learning Curve for the conventional PD (iteration $=1$ )

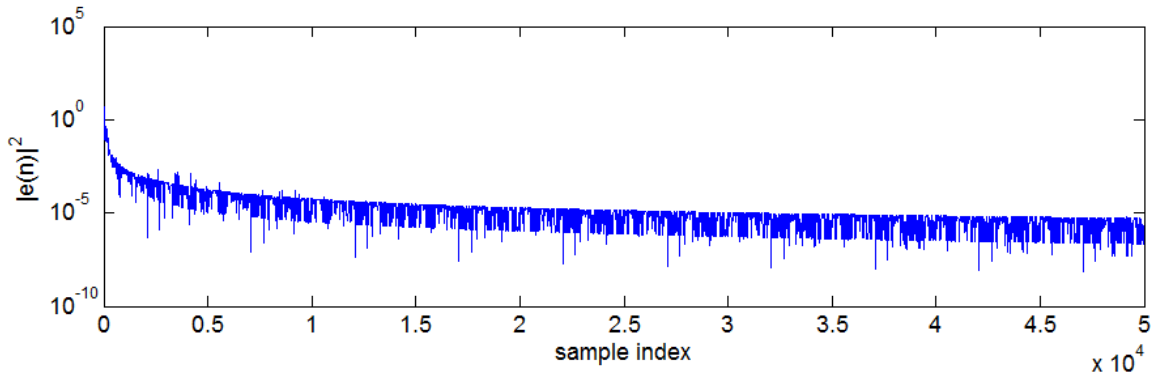

Figure 4. (b) Learning Curve for the proposed PD (iteration $=10$ )

Figure 5 shows the power spectrum at the PA output. The graphs represent as follows: (a) Tx signal; (b) PA output without DPD; (c) PA output with conventional DPD (max_iter $=1$ ) and 5,000 feedback samples $(\mathrm{N}=5,000)$; (d) PA output with proposed DPD $(\max$ iter $=10)$ and 5,000 feedback samples $(\mathrm{N}=$ 5,000); and (e) PA output with conventional DPD (max_iter $=1)$ and 170,000 feedback samples $(\mathrm{N}=$ 170,000). Comparing (c) and (d), it is seen that the proposed method performs much better than the conventional technique with the same feedback samples. The spectral regrowth is reduced by an additional $13 \sim 14 \mathrm{~dB}$ by using the proposed technique. Comparing (d) and (e), the proposed method shows nearly identical performance with the conventional DPD technique using 170,000 feedback samples, while the proposed method requires only 5,000 feedback samples. This result indicates that the proposed technique can relieve the requirement on the feedback memory size without linearization performance loss.

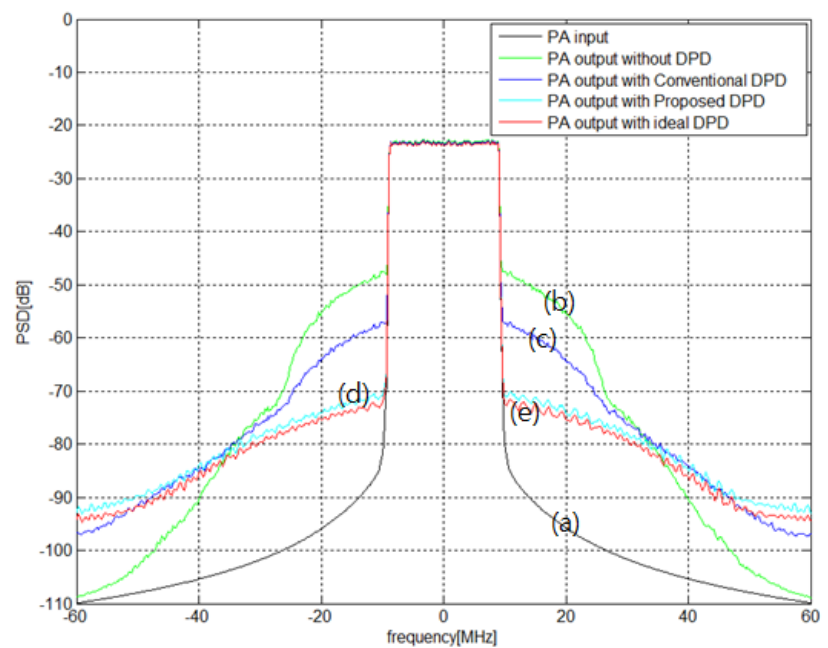

Figure 5. Spectrum comparison at PA output 


\section{CONCLUSION}

A polynomial DPD technique based on an iteration structure was proposed. Since the adaptive algorithm highly depends on the initial condition, it is possible to obtain performance using in short memory similar to that accomplished with long memory by keeping and iteratively using the converged DPD parameters at the former iteration as the initial condition at the current iteration. Computer simulation results show that a DPD technique that requires relatively short feedback samples can achieve linearization performance similar to that implemented using long memory.

\section{ACKNOWLEDGEMENTS}

This research was supported by the World Class 300 R\&D projects funded by the Small and Medium Business Administration of Korea (S2318109).

\section{REFERENCES}

[1] S.C. Cripps, Adavanced techniques in RF POWER AMPLIFIER DESIGN. Artech House, 2002.

[2] P.B. Kennington, High Linearity RF Amplifiers Design. Reading, MA: Artech House, 2000.

[3] Young-Doo Kim, Eui-Rim Jeong, and Yong H. Lee. "Adaptive compensation for power amplifier nonlinearity in the presence of quadrature modulation/demodulation errors", IEEE Transactions on Signal Processing, 55(9) (2007): 4717-4721.

[4] Chang Byong-Kun, Jeon Chang-Dae, Song Dong-Hyuk, "Performance Improvement in Alternate Mainbeam Nulling by Adaptive Estimation of Convergence Parameters in Linearly Constrained Adaptive Arrays", Journal of information and communication convergence engineering, pp.392-398, vol. 7, no. 3, 2009.

[5] Roman Marsalek, Pascale Jardin, and Geneviève Baudoin. "From post-distortion to pre-distortion for power amplifiers linearization", Communications Letters, IEEE 7(7) (2003): 308-310.

[6] Sungho Choi, Eui-Rim Jeong, and Yong H. Lee. "A direct learning structure for adaptive polynomial-based predistortion for power amplifier linearization", Vehicular Technology Conference, 2007. VTC2007-Spring. IEEE 65th. IEEE, 2007.

[7] A.A.M. Saleh, "Frequency-independent and frequency-dependent nonlinear models of TWT amplifiers", IEEE Trans. Commun., vol. COM-29, no. 11, (Nov 1981): 1715-1720.

\section{BIOGRAPHIES OF AUTHORS}

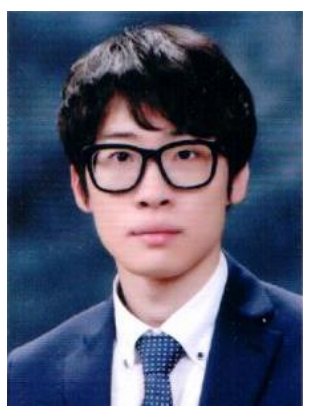

Kwang-Pyo Lee received the B.S. degree from the Department of Radio Wave Engineering at Hanbat National University, Daejeon, Korea, in 2014. He is currently pursuing the master's degree in the Department of Radio Wave Engineering at Hanbat National University, Daejeon, Korea. His research interests include the areas of digital signal processing, pre-distortion, and modem design.

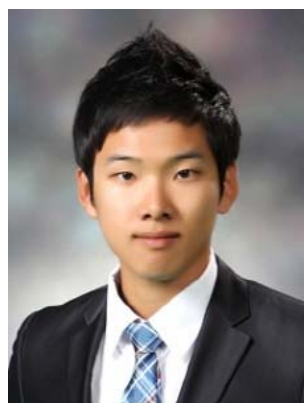

Soon-il Hong received the B.S. degree from the Department of Radio Wave Engineering at Hanbat National University, Daejeon, Korea, in 2014. He is currently pursuing the master's degree in the Department of Radio Wave Engineering at Hanbat National University, Daejeon, Korea. His research interests include the areas of digital signal processing, pre-distortion, and modem design. 


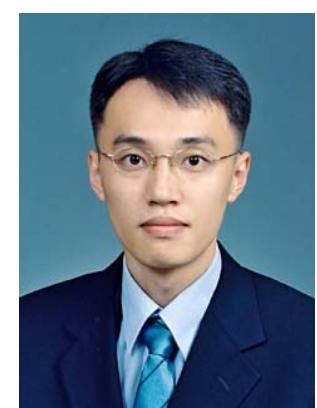

Eui-Rim Jeong received the B.S., M.S., and Ph.D. degrees from the Department of Electrical Engineering at the Korea Advanced Institute of Science and Technology (KAIST), Daejeon, Korea, in 1995, 1997, and 2001, respectively. He is currently an associate professor in the Department of Information and Communication Engineering, Hanbat National University, since 2009. His research interests include the areas of communication signal processing, pre-distortion, and modem design. 